\title{
A resonance Rayleigh scattering study of unlabeled and pyrene-labeled poly(acrylic acid) polymers
}

\author{
Telma Costa*, J. Seixas de Melo* \\ Department of Chemistry, University of Coimbra, 3004-535 Coimbra, Portugal
}

\section{A R T I C L E I N F O}

\section{Article history:}

Received 22 August 2008

Received in revised form 22 October 2008

Accepted 24 October 2008

Available online 6 November 2008

\section{Keywords:}

Resonance Rayleigh scattering

Poly(acrylic acid)

Pyrene

\begin{abstract}
A B S T R A C T
Three different unlabeled poly(acrylic acid) (PAA) polymers, with molecular weight of 2, 150 and $450 \mathrm{~kg} /$ $\mathrm{mol}$, and the correspondent PAA labeled with pyrene (PAAMePy polymers) were investigated, in aqueous solution and in dioxane: water mixtures, using the resonance Rayleigh scattering (RRS) technique. The pH showed to strongly affect the polymer conformation and a decrease of the scattering intensity $\left(I_{\mathrm{RRS}}\right)$ was observed, which is consistent with an increase of the hydrodynamic radius, reflecting an expanded polymer conformation in solution. The obtained $\mathrm{p} K_{\mathrm{a}}$ values were found higher for the labeled by comparison with the corresponding unlabeled polymers. For the PAAMePy polymers the increment of the water content in dioxane:water mixtures leads to an increase of the $I_{\text {RRS }}$ intensity; however, for the unlabeled PAA polymers non-significant differences were observed. In general, for the fluorescently labeled polymers, a good agreement between the present RRS results and those previously obtained from fluorescence measurements could be found.
\end{abstract}

(c) 2008 Elsevier B.V. All rights reserved.

\section{Introduction}

Polymers in general and hydrophobically modified polymers (HMP), in particular, are of major importance from industrial and academic points of view. Their interaction with surfactants, are frequently used in pharmaceutical formulations [1,2], cosmetics [1,2] and in all modern water based paints [3,4]. From a pure academic point of view their conformation and the complex formation with other amphiphilic molecules, such as surfactants and cyclodextrins, is currently gaining a renewed interest.

Fluorescence has shown to be a powerful tool to investigate this kind of systems. The photophysical behavior of poly(acrylic acid) randomly labeled with pyrene, with different molecular weights and degrees of labeling, has been extensively studied through absorption and fluorescence techniques [5,6]. Pyrene, due to its unique characteristics, long singlet lifetimes, excimer formation, vibronic structure dependence with polarity [7-9], has a widespread use as a unique probe in the colloidal domain. One of the most useful relations is the excimer-to-monomer ratio $\left(I_{\mathrm{E}} / I_{\mathrm{M}}\right)$, which can be directly related with the dynamic behavior of the polymer in solution. For a negatively charged polyelectrolyte, it was shown that by increasing the $\mathrm{pH}$, the polymer conformation changes from a compact to an expanded coil $[5,6]$. The $I_{\mathrm{E}} / I_{\mathrm{M}}$ ratio variation has also showed to be very useful to detect the complex formation between the PAAMePy polymers and cyclodextrins

\footnotetext{
* Corresponding authors. Tel.: +351 239854463; fax: +351 239827703.

E-mail addresses: tcosta@qui.uc.pt (T. Costa), sseixas@ci.uc.pt (J. Seixas de Melo).
}

$[10,11]$. Additionally, the vibronically resolved structure of the monomer emission-the ratio between the first and third vibronic band of the monomer emission, the $I_{1} / I_{3}$ ratio-exhibits high sensitivity to changes in the polarity of the local environment felt by the pyrene chromophore and has also been extensively used as a polarity scale [12-18].

Nevertheless, several techniques, besides fluorescence, can be used to study polymer conformation and complex formation, such as conductivity, [19,20] light scattering, [21] NMR, [19,20], etc. Resonance Rayleigh scattering (RRS) is a relatively new technique that has been successfully employed in the study of macromolecular and biological systems. When light interacts with molecules (polymers or chromophores) it can be absorbed or scattered. The scattering process occurs due to the differences in the refractive index of the molecule and the solvent $[22,23]$. The difference between light scattering and RRS techniques lies in the scattering wavelength; in the case of the light scattering technique, the measurement is performed away from the absorption band whereas in the case of RRS the scattering is observed at, or close to, the molecular absorption band. In the RRS technique the frequency of the absorbed electromagnetic wave is equal to the scattering frequency. Due to the high absorption of light energy by the electron, light will be re-scattered. An increase of the scattering intensity is then observed, constituting the basis of the RRS technique. Among other factors, this increase depends on the molar absorption coefficient $[23,24]$ and on the volume of the scattering object [23]. Examples of applications of RRS can be found in the study of the interaction of heparin with diphenyl naphthylmethane dyes [24] and anthra- 
cycline antibiotics with surfactants [25]. The technique was also successfully applied to the determination of the critical aggregation concentration (cac) of proteins induced by $\mathrm{Al}(\mathrm{III})$ [26] and of surfactants in the absence and presence of $\beta$-cyclodextrin [27]; and on the interaction between poly(acrylic acid) and SDS and CTAB surfactants [28].

\section{Experimental}

\subsection{Polymers}

The unlabeled poly(acrylic acid) polymers with nominal weight $M_{\mathrm{n}}=2000,150,000$ and 450,000 g/mol (Aldrich) were used as received. Their acronyms are based on their molecular weight, i.e., PAA(2), PAA(150) and PAA(450), where 2, 150 and 450 stands for the $2000 \mathrm{~g} / \mathrm{mol}, 150,000 \mathrm{~g} / \mathrm{mol}$ and $450,000 \mathrm{~g} / \mathrm{mol}$ polymers, respectively. The PAA polymers randomly labeled with 1-pyrenylmethylaminehydrochloride (Aldrich) were synthesized according to the procedure described elsewhere $[5,6]$. All reagents and solvents used in the synthesis were obtained from commercial sources and used as received. The pyrene (Py) labeling content was determined by UV spectroscopy by comparison with the parent compound 1-pyrenylmethylamine $\left(\epsilon=37070 \mathrm{M}^{-1} \mathrm{~cm}^{-1}\right.$ in methanol). The nomination of the polymers was again based on the molecular weight of the PAA polymer and on the Py content of the polymers. The polymers here investigated are denoted as PAAMePy(2)52, PAAMePy(150)55 and PAAMePy(450)53 where 52,55 or 53 corresponds to number of PAA monomer units per Py chromophore, respectively (Scheme 1).

The solvents used in the polymer solutions were of spectroscopic or equivalent grade. Water used for the polymer solutions was twice distilled and passed through a Millipore apparatus. The measured $\mathrm{pH}$ values were obtained with a Crison micropH 2000 and adjustments of the hydrogen ion concentration of the solutions were made with dilute $\mathrm{HCl}$ and $\mathrm{NaOH}$ solutions.

\subsection{Experimental techniques}

The fluorescence and RRS spectra were recorded with a HoribaJobin-Ivon SPEX Fluorog 3-22 spectrometer and were corrected for the instrumental response. The Fluorolog consists in a modular spectrofluorimeter with double grating excitation (range 200$950 \mathrm{~nm}$, optimized in the UV and with a blazed angle at $330 \mathrm{~nm}$ ) and emission (range $200-950 \mathrm{~nm}$, optimized in the visible and with a blazed angle at $500 \mathrm{~nm}$ ) monochromators. The excitation source consists of a ozone-free $450 \mathrm{~W}$ Xenon lamp and the emission detector is an Hamamatsu R928 Photomultiplier (200$900 \mathrm{~nm}$ range), cooled with a Products for Research thermoelectric refrigerated chamber (model PC177CE005), or a Hamamatsu R5509-42 (900-1400 nm range), cooled to $193 \mathrm{~K}$ in a liquid nitrogen chamber (Products for Research model PC176TSCE-005-) and a photodiode as the reference detector. For the RRS experiments, the

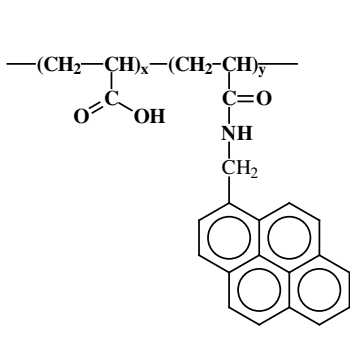

$$
\begin{aligned}
& \text { PAA: } \mathbf{2} \text { kg/mol } \\
& \left.\begin{array}{l}
\mathrm{x}=0.981 \\
\mathrm{y}=0.019
\end{array}\right\} \text { PAAMePy(2)52 } \\
& \text { PAA: } 150 \mathrm{~kg} / \mathrm{mol} \\
& \left.\begin{array}{l}
x=0.98 \\
y=0.02
\end{array}\right\} \text { PAAMePy (150)55 } \\
& \text { PAA: } 450 \mathrm{~kg} / \mathrm{mol} \\
& \left.\begin{array}{l}
x=0.982 \\
y=0.018
\end{array}\right\} \text { PAAMePy(450)53 }
\end{aligned}
$$

Scheme 1. excitation and emission spectra were recorded in the 200-700 nm range with synchronous scanning at $\lambda_{\mathrm{ex}}=\lambda_{\mathrm{em}}$ (i.e., $\Delta \lambda=0 \mathrm{~nm}$ ); the slit widths are of $2.0 \mathrm{~nm} / 2.0 \mathrm{~nm}(\mathrm{Ex} / \mathrm{Em})$ and $3.0 \mathrm{~nm} / 2.0 \mathrm{~nm}(\mathrm{Ex} /$ $\mathrm{Em})$ for the labeled and unlabeled systems, respectively.

\section{Results and discussion}

\subsection{Influence of the molecular weight of the PAAMePy polymers on the RRS spectra}

\subsubsection{Aqueous solutions}

Three different molecular weight poly(acrylic acid) polymers $(2000,150,000$ and $450,000 \mathrm{~g} / \mathrm{mol})$ labeled with pyrene, PAAMe$\operatorname{Py}(2) 52$, PAAMePy(150)55 and PAAMePy(450)53, are the subject of the current investigation. The RRS spectra of the PAAMePy polymers in aqueous solution are presented in Fig. 1. From the spectra in Fig. 1 five peaks at $\sim 294, \sim 304, \sim 318, \sim 336$ and $\sim 376 \mathrm{~nm}$ can be observed, and shown to be independent of the molecular weight of the PAA polymer. The absorption spectra of the pyrene-labeled polymers display the characteristic vibronically resolved spectra of this chromophore but are red-shifted $(8-10 \mathrm{~nm})$ relative to pyrene itself (see the inset in Fig. 1 for an example of the absorption spectra of pyrene-labeled polymer).

For a polymer concentration of $0.1 \mathrm{~g} / \mathrm{l}$, the intensity of the peak at $375 \mathrm{~nm}$ is approximately constant and independent of the molecular weight of the PAA polymer. However, the peak with a maximum scattering wavelength at $\sim 294 \mathrm{~nm}$ significantly increases upon going from the low $\left(M_{\mathrm{w}}=2000 \mathrm{~g} / \mathrm{mol}\right)$ to the high $\left(M_{\mathrm{w}}=450000 \mathrm{~g} / \mathrm{mol}\right)$ molecular weight PAA polymer, showing that by increasing the polymer $M_{\mathrm{w}}$ an increase of the RRS scattering intensity, $I_{\mathrm{RRS}}$, is observed. Indeed, the excess of Rayleigh ratio promoted by the concentration fluctuations in a solution of small independent particles $\left(n \sim n_{0}\right)$ is given by the following equation [29]

$\Delta R_{\theta}=\left[\frac{4 \pi^{2} n_{0}^{2}(\partial n / \partial c)}{N_{\mathrm{A}} \lambda^{4}}\right] \cdot M c=K M c$

where $\partial n / \partial c$ is the refractive index increment, $M$ is the molar mass, $N_{\mathrm{A}}$ is Avogadro's number, $\lambda$ is the wavelength of light, $K$ is the optical constant and $c$ the concentration. Thus, in a diluted solution the increase of the molecular weight of the solute, or in this case of the polymer, leads to an increase of the scattered light and consequently of its intensity.

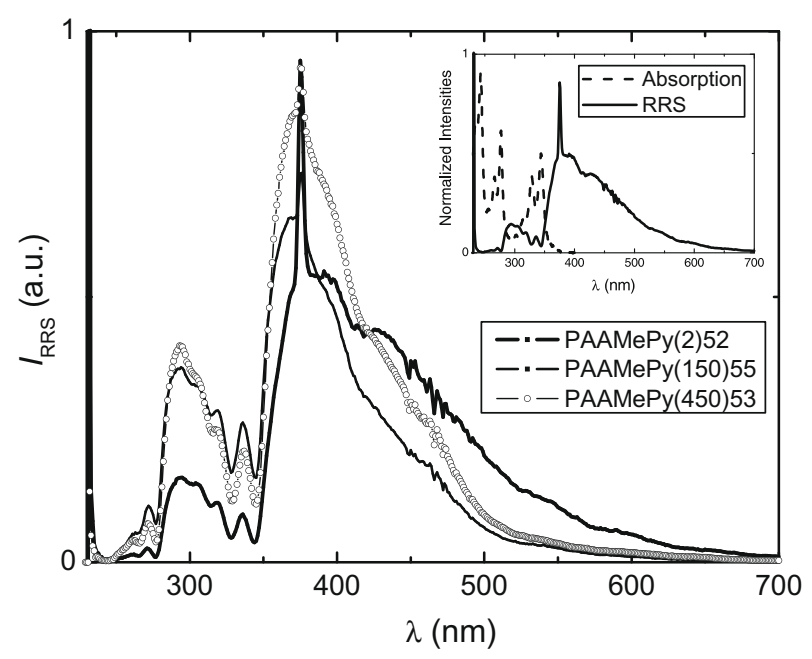

Fig. 1. RRS spectra of the PAAMePy(2)52, PAAMePy(150)55 and PAAMePy(450)53, at $\mathrm{pH}$ 3. As inset is shown the absorption and the RRS spectra of the PAAMePy $(2) 52$ polymer, at $\mathrm{pH} 3$. 
Fig. 2A shows the effect of the $\mathrm{pH}$ on the RRS spectra of the PAAMePy(150)55 polymer. A general decrease of the $I_{\mathrm{RRS}}$ is observed with the $\mathrm{pH}$; however, the ratio between the maximum scattering intensity and the other peak intensities also varies with pH, Fig. $2 \mathrm{~A}$ (inset), suggesting that this ratio is probing the conformational changes suffered by the polymer. The dependence of the ratio between the intensities at $\lambda \sim 294 \mathrm{~nm}$ and $\lambda \sim 375 \mathrm{~nm}$, defined as $I_{\mathrm{B}} /$ $I_{\mathrm{F}}(R)$ is for the PAAMePy(2)52, PAAMePy(50)55 and PAAMePy(450)53 polymers, presented in Fig. 2B. For the long $M_{\mathrm{w}}$ polymers [PAAMePy(150)55 and PAAMePy(450)53] a decrease of the $I_{\mathrm{B}} / I_{\mathrm{F}}$ ratio with the $\mathrm{pH}$, which levels on up to $\mathrm{pH} \sim 7$, is observed. The use of an intensity ratio instead of the absolute RRS intensity at a given and particular wavelength $\left(I_{\mathrm{RRS}}\right)$ constitutes an internal correction that was introduced to avoid some of the dispersion that occurs in the RRS intensity values, due to slightly different experimental conditions: e.g., concentration, $\mathrm{pH}$, temperature, etc., which could not be avoided if, instead, we had only considered the $I_{\mathrm{RRS}}$ signal.

At a given and specific wavelength the RRS intensity depends on the absorption at this same wavelength, i.e., to a maximum absorption value usually corresponds a higher scattering intensity [23]. For a constant absorption value (concentration), the RRS technique can be used to follow the conformational changes of the polymer chain. The relationship between the RRS intensity and the size of the polymer chain was recently described by Li et al. [28] which showed that the RRS intensity is reciprocally proportional to the hydrodynamic radius $\left(R_{\mathrm{H}}\right)$ of the molecule. Both $I_{\mathrm{RRS}}$ and $I_{\mathrm{B}} / I_{\mathrm{F}}$ ratio decrease with the increasing $\mathrm{pH}$; this decrease reflects the augment of the $R_{\mathrm{H}}$ for the long size chain PAAMePy polymers at high $\mathrm{pH}$ values. At low $\mathrm{pH}$, the pyrene-pyrene interactions, within the
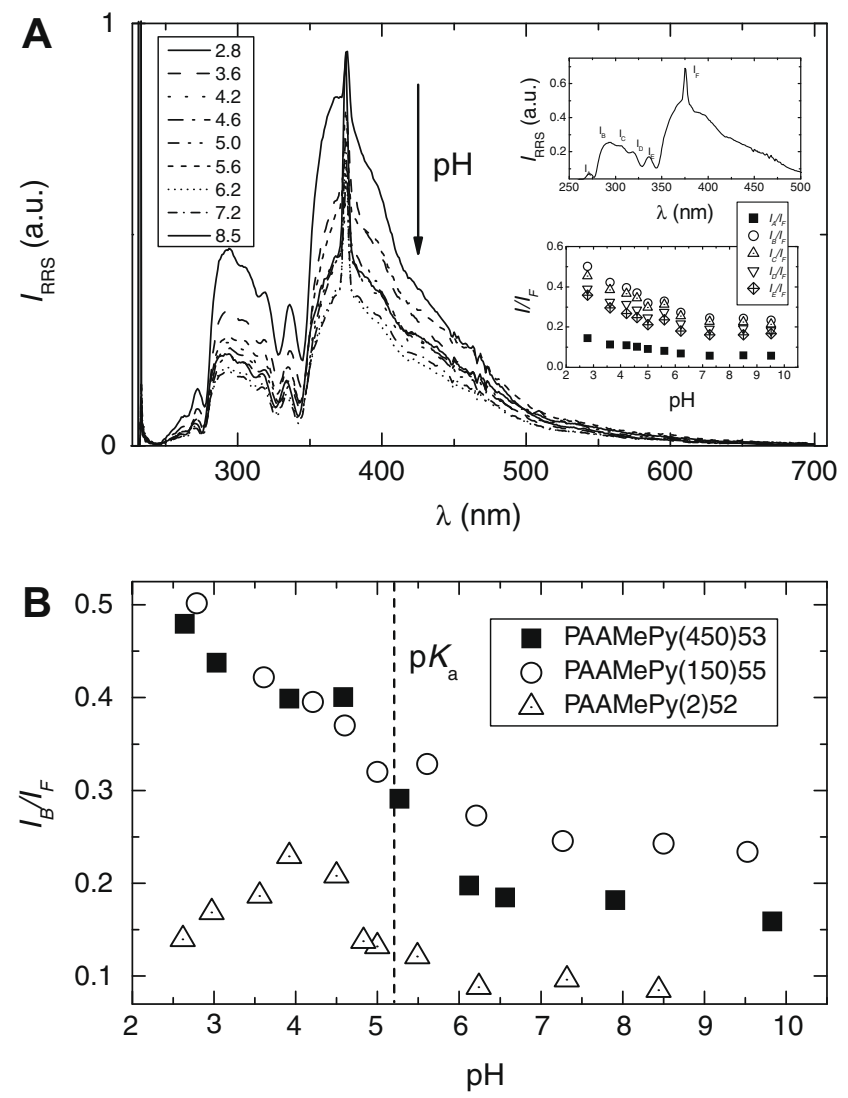

Fig. 2. (A) RRS spectra at different $\mathrm{pH}$ values for the PAAMePy(150)55 polymer, $c=0.1 \mathrm{~g} / \mathrm{l}$. Shown as inset are the $I_{\mathrm{A}} / I_{\mathrm{F}}, I_{\mathrm{B}} / I_{\mathrm{F}}, I_{\mathrm{C}} / I_{\mathrm{F}}, I_{\mathrm{D}} / I_{\mathrm{F}}$ and $I_{\mathrm{E}} / I_{\mathrm{F}}$ ratios as a function of the $\mathrm{pH}$. (B) Dependence of the $I_{\mathrm{B}} / I_{\mathrm{F}}$ ratio obtained from the RRS spectra on the $\mathrm{pH}$, for three different polymers: PAAMePy(2)52, PAAMePy(150)55 and PAAMePy $(450) 53$.
PAA chain, lead to the adoption of a coiled conformation (high $I_{\mathrm{RRS}}$ and $I_{\mathrm{B}} / I_{\mathrm{F}}$ values) $[5,6]$. The increase of the $\mathrm{pH}$ leads to the progressive ionization of the carboxylic groups and the electrostatic repulsion between the carboxylate groups competes with the hydrophobic interactions between adjacent pyrene groups $[5,6]$. At alkaline $\mathrm{pH}$ values the former forces become dominant and an expansion of the PAA chain is then observed which is mirrored by the decrease of the $I_{\text {RRS }}$ and $I_{\mathrm{B}} / I_{\mathrm{F}}$ ratio values. Indeed, similar conclusions were previously drawn from fluorescence studies $[5,6]$. As stated in the experimental section, the adjustments of the $\mathrm{pH}$ were made with dilute $\mathrm{HCl}$ and $\mathrm{NaOH}$ solutions. In previous fluorescence studies on a similar PAAMe polymer labeled with naphthalene [30] the dependence of the excimer-to-monomer ratio $\left(I_{\mathrm{E}} / I_{\mathrm{M}}\right)$ ratio with the $\mathrm{pH}$ was performed with two different acids $\left[\mathrm{HCl}\right.$ and perchloric acid $\left.\left(\mathrm{HClO}_{4}\right)\right]$, showing that the same effect was obtained with different counterions and that these do not contribute significantly to the change in the polymer conformation. Consequently, it is not likely that the observed changes in the RRS spectra result from the effect of the chlorine ions on the conformation adopted by the polymer coil, as they did not for analogous polymers.

The dependence of the $I_{\mathrm{B}} / I_{\mathrm{F}}(=R)$ ratios with the $\mathrm{pH}$ presented in Fig. 2B, were fitted to a sigmoidal Boltzman-like equation according to the following equation [5]

$R=I_{0}+\frac{I_{1}-I_{0}}{1+e^{\frac{p H-p K_{a}}{d p H}}}$

from which the $\mathrm{p} K_{\mathrm{a}}$ could be estimated. The obtained values, for the PAAMePy(150)55 and PAAMePy(450)53 polymers, were, respectively, $\sim 5.1$ and $\sim 5.2$. From previous spectral and photophysical investigations on the PAAMePy(150)55 [6] and PAAMePy(450)53 [5] polymers the determination of the $\mathrm{p} K_{\mathrm{a}}$ values of these polymers could be obtained from the peak-to-valley $\left(P_{\mathrm{A}}\right)$ ratio of the $\mathrm{S}_{0} \rightarrow \mathrm{S}_{2}$ absorption band and found to be, respectively, 5.8 and 5.5. From these results it can be seen that a good agreement between the values obtained from absorption and RRS techniques could be obtained.

In the case of the PAAMePy(2) polymers, a different behavior was found. As can be seen from Fig. $2 \mathrm{~B}$, there is first an increase of the $I_{\mathrm{B}} / I_{\mathrm{F}}$ ratio with $\mathrm{pH}$ until $\mathrm{pH} \sim 4$, and only above this value the $I_{\mathrm{B}} / I_{\mathrm{F}}$ ratio progressively decreases to the initial values. This unusual feature was also observed with the dependence of the $I_{\mathrm{E}} /$ $I_{\mathrm{M}}$ ratio with the $\mathrm{pH}$, where the $I_{\mathrm{E}} / I_{\mathrm{M}}$ ratio increases up to values close to the $\mathrm{p} K_{\mathrm{a}}$ value, followed by a slight decrease of the $I_{\mathrm{E}} / I_{\mathrm{M}}$ ratio [5]. The initial increase of the $I_{\mathrm{B}} / I_{\mathrm{F}}$ ratio (and $I_{\mathrm{E}} / I_{\mathrm{M}}$ ratio) [5] can be explained by a contraction of the PAAMePy(2)52 polymer chain, i.e., the pyrene-pyrene interactions are promoted by the introduction of some charges in the system and the polymer adopts a "micelle-like" conformation where the (essentially two) pyrenes are located [5]. A further increase of the alkalinity of the media, leads to a predominance of the (repulsive) electrostatic forces with an expansion of the polymer chain with the consequent loss of the "micelle-like" structure. In the case of the short chain polymer the inflexion in the $I_{\mathrm{B}} / I_{\mathrm{F}}$ ratio with $\mathrm{pH}$ is found between $\mathrm{pH} 4$ and 5 , which is, within the experimental error, in very good agreement with the maximum observed in the $I_{\mathrm{E}} / I_{\mathrm{M}}$ ratio as a function of the $\mathrm{pH}$ [5]. However, it should be stressed that the $I_{\mathrm{B}} / I_{\mathrm{F}}$ ratio is not related with the monomer-excimer kinetics, i.e., with the $I_{\mathrm{E}} / I_{\mathrm{M}}$ ratio. The $I_{\mathrm{E}} / I_{\mathrm{M}}$ and $I_{\mathrm{B}} / I_{\mathrm{F}}$ are two different ratios (obtained from different experiments) that give complementary information on the conformational changes of the pyrene-labeled polymers submitted to different external factors. It is also worth noting that the $I_{\mathrm{B}} / I_{\mathrm{F}}$ ratio is not present in the unlabeled PAA polymers.

Another important observation is the fact that when the wavelength of Rayleigh scattering is located at, or close to, the absorp- 
tion band, the scattering intensity is enhanced by several orders of magnitude and the scattering intensity, that is, the resonance Rayleigh scattering (RRS) intensity, deviates from the Rayleigh law $\left(I \propto 1 / \lambda^{4}\right)[24]$. For this reason, the RRS spectra is also influenced by the different electronic transitions [24]; consequently, several components contribute to the observed spectra, namely the absorption and fluorescence of the chromophore, the solvent and also the overlap between the excitation and emission spectra [31]. In our study all the RRS spectra were corrected for the instrumental response of the equipment (such as the Xe lamp profile, monochromator and detector response, etc.); however, as can be seen from the inset in Fig. 1, and particularly in the wavelength region between 250 and $400 \mathrm{~nm}$, the RRS bands clearly results from the contributions of the pyrene chromophore absorption and fluorescence (the peak at $\sim 376 \mathrm{~nm}$ corresponds to the maximum emission wavelength of the pyrene monomer). However, if one observes the red region of the spectra $(>385 \mathrm{~nm}$, outside the absorption and emission region of the pyrene chromophore) a decrease of the RRS intensity with the incident wavelength is clearly seen. Hence, we performed the correction of the obtained RRS spectra by taking into account the solvent contribution and we neglected the intensity values below $380 \mathrm{~nm}$. The $I_{\text {RRS }}$ obtained at $385 \mathrm{~nm}$ is plotted as a function of the $\mathrm{pH}$, and it is presented in Fig. 3. From the normalization of the $I_{\text {RRS }}$ intensity at $385 \mathrm{~nm}$ with the $I_{\mathrm{B}} / I_{\mathrm{F}}$ ratio, it can be seen that they are in agreement with one another. A clear indication of the two conformational regimes (coil and expanded) is present in both data, proving that the $I_{\mathrm{B}} / I_{\mathrm{F}}$ ratio (which includes the contributions from fluorescence and absorption processes) can also be used to monitor the changes of the polymer conformational changes.

\subsubsection{Dioxane:water mixtures}

In dioxane:water mixtures significant differences, relative to the situation in water, were observed in the absorption and emission spectra of the PAAMePy polymers. The PAAMePy polymers are amphiphilic molecules and in aqueous solution they can orient themselves in a way that they expose their hydrophilic groups toward water and simultaneously protect their hydrophobic (pyrene) groups from the contact with water and, consequently, they are able to form hydrophobic microdomains. Dioxane is a good solvent to the pyrene groups being completely miscible with water. Therefore, the presence of dioxane in an aqueous solution significantly increases the quality of the solvent to the hydrophobic probe. The pyrene-solvent contacts become more favorable and the polymer adopts a structure of a random coil, which can be mirrored by a decrease in the intramolecular interactions occurring between the pyrene groups (decrease of the $I_{\mathrm{E}} / I_{\mathrm{M}}$ ratio). A decrease of the overall scattering intensity (Fig. $4 \mathrm{~A}$ ) and of the $I_{\mathrm{B}} / I_{\mathrm{F}}$ ratio (Fig. 4B) was also observed for the long chain polymers.

In the case of the short-size chain polymer, PAAMePy(2)52, a slight decrease of the $I_{\mathrm{B}} / I_{\mathrm{F}}$ ratio was also observed; however, the decrease of the $I_{\mathrm{B}} / I_{\mathrm{F}}$ ratio and of the overall $I_{\mathrm{RRS}}$ is not as high as in the case of the long-sized chain polymers labeled with pyrene. The reason for the less abrupt decrease on the scattering intensity lies, not only on the lower molecular weight of the polymer (minor changes in the $R_{\mathrm{H}}$ ), but also, and essentially, in the fact that $59 \%$ of the PAAMePy(2)52 chains are unlabeled, $31 \%$ chains are single-labeled and only $8.4 \%$ and $1.6 \%$ chains possess two and three pyrene units, respectively [5]. As a consequence, the gradual increment of the good solvent contribution has a small effect on the unlabeled or single-labeled polymer chains. It is likely that only the PAAMePy(2)52 polymer chains with two or more pyrene groups will show a detectable change in their conformation in solution. The dependence of the $I_{\mathrm{E}} / I_{\mathrm{M}}$ ratio with the molar fraction of dioxane showed, however, a different behavior. Indeed, the excimer emission reaches a maximum at a molar fraction of dioxane $\left(x_{\mathrm{Dx}}\right)$ equal to 0.083 decreasing (with the increase in $x_{\mathrm{Dx}}$ ) from thereon. The initial increase in the $I_{\mathrm{E}} / I_{\mathrm{M}}$ ratio is probably due to the existence of van der Waals forces between the pyrene groups. This increase, of the intramolecular interactions between the pyrene groups of the PAAMePy(2)52 polymer, was not detected through the RRS spectra due to the fact that no significant changes in the polymer conformation could be observed. The increase of the $I_{\mathrm{E}} / I_{\mathrm{M}}$ ratio is mainly promoted by small movements of the PAA chain and not by a contraction (decrease of the $R_{\mathrm{H}}$ ) of the polymer. Up to $x_{\mathrm{Dx}} \sim 0.083$, the dioxane content in solution is high and favorable for the pyrene probe to overcome the contributions from the van der Waals interactions between the pyrene groups, consequently leading to a decrease of the pyrene-pyrene interactions, i.e., to an expansion of the polymer chain. A parallel behavior was found with the analogous polymer $\left(M_{\mathrm{w}}=150 \mathrm{~kg} / \mathrm{mol}\right)$ randomly labeled with naphthalene, $\mathrm{Np}$, in organic solvent:water mixtures, [32] in aqueous solution [30] and in the presence of surfactants [33].

\subsection{Influence of the labeling on the RRS spectra}

Being the PAAMePy polymers randomly labeled with pyrene, they allow the study of the dynamics of the polymer in a situation where only intramolecular interactions are found to be present.
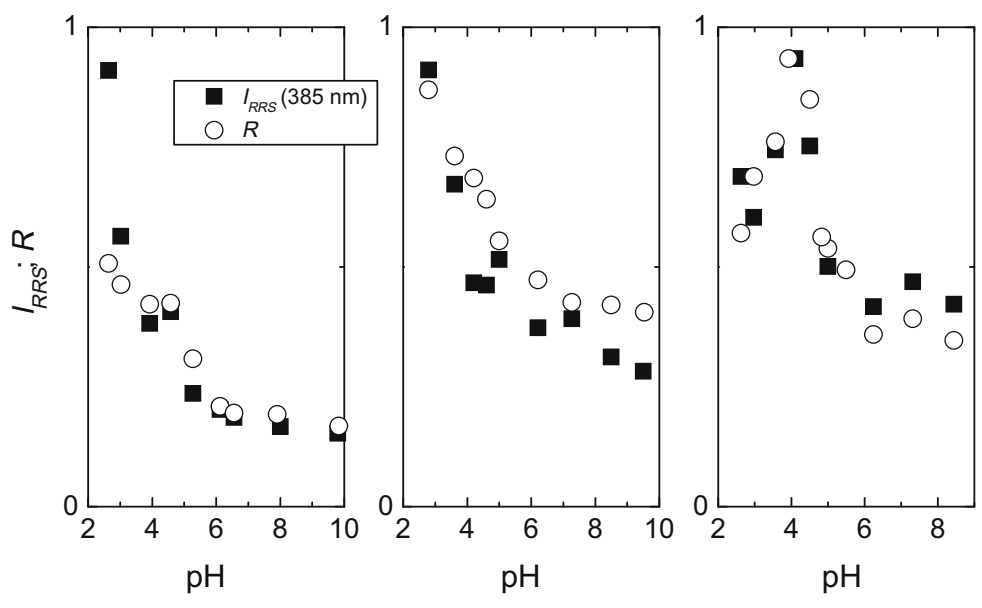

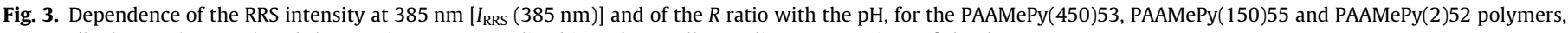
$c=0.1 \mathrm{~g} / \mathrm{l}$. The $I_{\mathrm{RRS}}(385 \mathrm{~nm})$ and the $R$ ratio were normalized in order to allow a direct comparison of the data. 

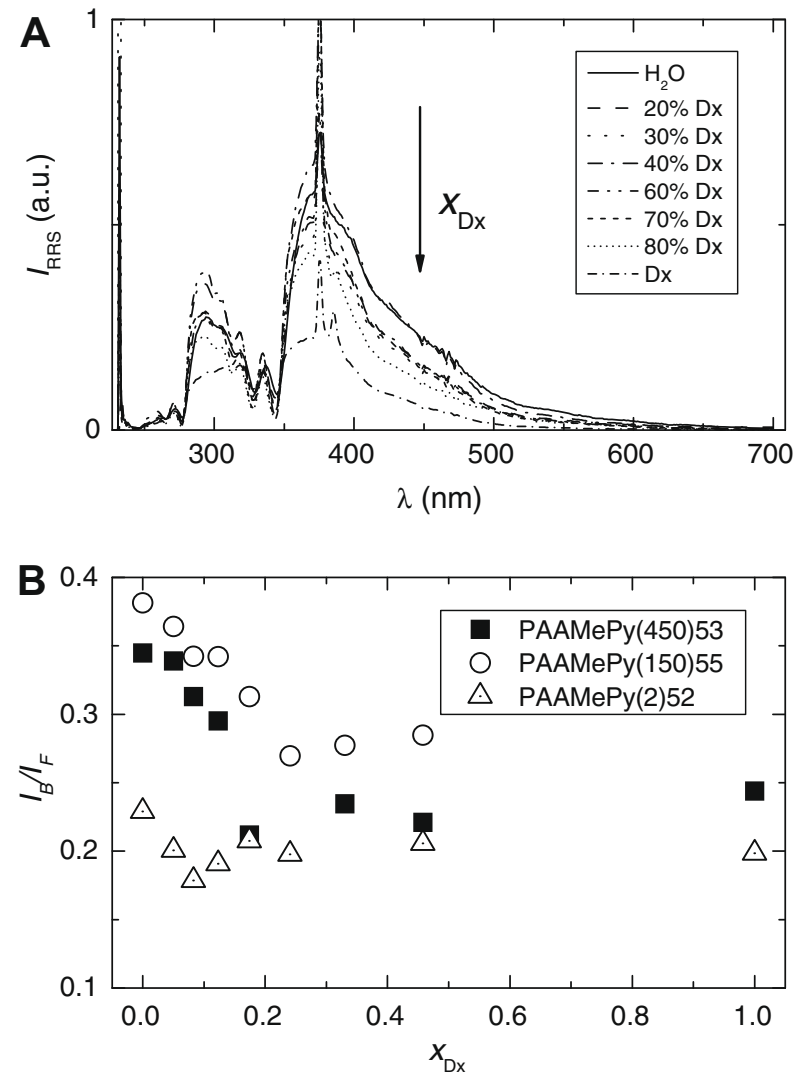

Fig. 4. RRS spectra of the PAAMePy(450)53 polymer in dioxane:water mixtures, $c=0.1 \mathrm{~g} / \mathrm{l}$. Dependence of the $I_{\mathrm{B}} / I_{\mathrm{F}}$ ratio obtained from the RRS spectra on the molar fraction of dioxane, for three different polymers: PAAMePy(2)52, PAAMePy(150)55 and PAAMePy(450)53.

The RRS technique can be used to follow the conformational changes of the PAA polymers with and without labeling.

In this section, we wish to investigate the effect of the probe (labeling) on the PAA conformation in solution as seen by RRS. Fig. 5 shows the RRS spectra of three different poly(acrylic acid) polymers: $\mathrm{PAA}(2), \mathrm{PAA}(150)$ and $\mathrm{PAA}(450)$. Comparison of the unlabeled with the correspondent labeled PAA polymers (presented in Fig. 1), shows that the introduction of pyrene groups onto

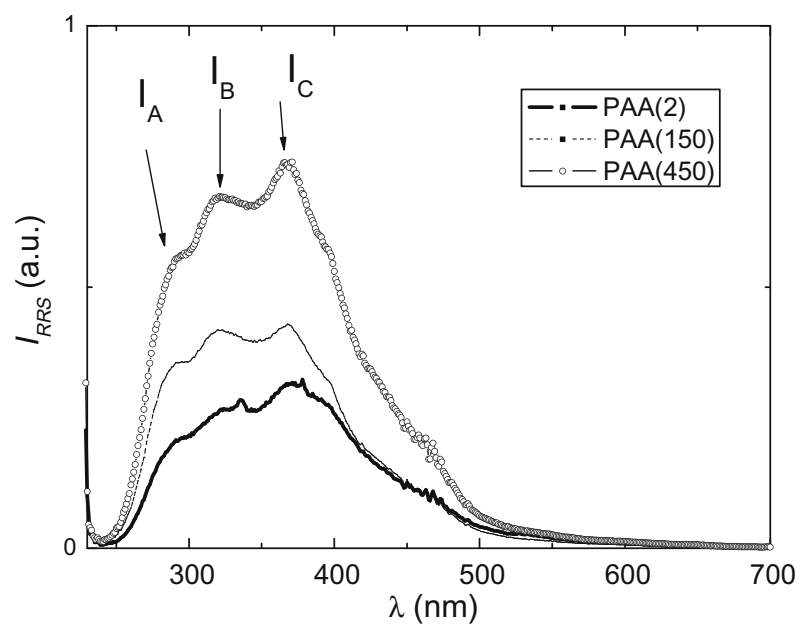

Fig. 5. RRS spectra of $\operatorname{PAA}(2), P A A(150)$ and $P A A(450)$ polymers in aqueous solution, at $\mathrm{pH} 3.5$ and $c=0.1 \mathrm{~g} / \mathrm{l}$. the PAA chain promotes significant differences in the RRS spectra. Indeed, the RRS spectra of the PAAMePy polymers corresponds to the RRS pattern found for the pyrene probe itself (data not shown). Contrary to what was observed in the RSS spectra of the Py labeled PAA polymers, where five peaks were found, for the unlabeled PAA polymers only three relevant peaks, with maximum intensities at $292\left(I_{A}\right), 320\left(I_{B}\right)$ and $366 \mathrm{~nm}\left(I_{C}\right)$, could be observed. Li et al. [28] have recently performed a study on the influence of the $\mathrm{pH}$ on the RRS spectra of a similar unlabeled PAA polymer $\left(M_{\mathrm{w}}=240,000 \mathrm{~g} / \mathrm{mol}\right)$ using the RRS technique. The RRS spectra, obtained for that polymer, showed a maximum scattering wavelength at $401 \mathrm{~nm}$, with two less intense scattering peaks at 471 and $536 \mathrm{~nm}$, respectively, which is in contrast with the RRS spectra shown in Fig. 5. Also from the data presented in Fig. 5, the shape of the RRS spectra should be independent of the molecular weight of the PAA polymer. This difference could again be potentially explained by the different experimental conditions, namely on the correction factors introduced, such as the monochromators bandpass, Xe lamp profile, etc. [31] on the RRS spectra.

The comparison of the $\mathrm{pH}$ and solvent effect on the PAA and PAAMePy polymers will be here presented, as an illustrative example, for the high molecular weight PAA(450) and PAAMePy(450)53 polymers, since the same behavior was found with the other investigated polymers. The changes in the PAA $(450)$ polymer will be presented in terms of the ratio between the intensity at $320\left(I_{\mathrm{B}}\right)$ and $366 \mathrm{~nm}\left(I_{C}\right), I_{B} / I_{C}$ ratio, since these two seem to show the more straightforward trends with $\mathrm{pH}$.

Fig. 6 shows the dependence of the $I_{\mathrm{B}} / I_{\mathrm{C}}$ and $I_{\mathrm{B}} / I_{\mathrm{F}}$ ratios as a function of the $\mathrm{pH}$ for, respectively, the unlabeled $\mathrm{PAA}(450)$ and for the correspondent pyrene-labeled polymer [PAAMePy(450)53]. In the two cases, a decrease of the $R$ ratio is observed, consistent with an expansion of the polymer chain. A more pronounced effect is observed with the pyrene-labeled PAA chain due to the presence of attractive hydrophobic interaction, within the PAAMePy(450)53 polymer, leading to a more compact coil at acidic $\mathrm{pH}$ values. A decrease of the $I_{B} / I_{C}$ ratio was also found for the PAA(2) polymer (not shown). Comparison between the labeled, $\operatorname{PAA}(2)$, and the unlabeled, PAAMePy(2)52, polymers (Fig. 2B) shows that in the case of these short-size chain polymers, the presence of the probe affects the behavior of the PAA chain in solution. This shows, as stated above, that the intramolecular interactions between the pyrene groups are responsible for a higher level of contraction of the polymer, observed at acidic pH values. When the attractive hydropho-

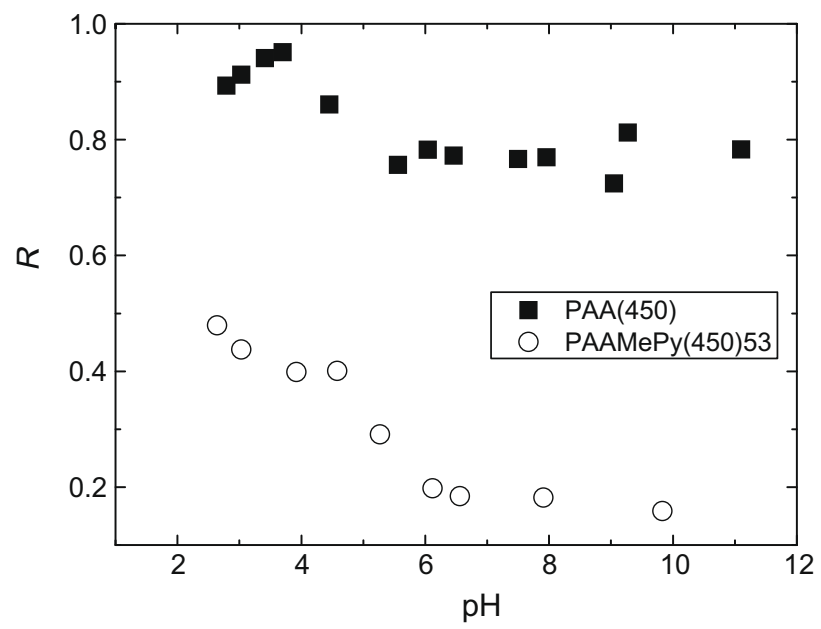

Fig. 6. Plot of the $R$ ratio as a function of the $\mathrm{pH}$ for the $\mathrm{PAA}(450)$ and PAAMePy $(450) 53$ polymers. The $R$ ratio consists in the $I_{\mathrm{B}} / I_{\mathrm{C}}$ and the $I_{\mathrm{B}} / I_{\mathrm{F}}$ ratios for the PAA(450) and PAAMePy(450)53 polymers, respectively. 
bic interactions, within the polymer chain, are absent, the short chain polymers behave as normal polyelectrolytes.

The fit of the dependence of the $I_{B} / I_{C}$ ratio with the $\mathrm{pH}$, using Eq. (2), allows the determination of the $\mathrm{p} K_{\mathrm{a}}$ values for the PAA polymers. The obtained value is $\sim 4.6$ for the $\operatorname{PAA}(150)$ and $\operatorname{PAA}(450)$ polymers, in agreement with the literature values (ca. 4.7 at $25^{\circ} \mathrm{C}$ ) [34]. For the unlabeled polymers, the $\mathrm{p} K_{\mathrm{a}}$ values are lower than those of the correspondent labeled polymers, thus showing that the introduction of the pyrene probe increases the apparent $\mathrm{p} K_{\mathrm{a}}$ of the PAA polymer. The hydrophobic interaction, between the pyrene groups, leads to a more compact conformation than that adopted by the unlabeled polymer. The potentiometric curves obtained for the labeled polymers [5] have a similar behavior to that found with poly(methacrylic acid), $[35,36]$ but much less intense in the former case. In the case of poly(methacrylic acid) the anomalous behavior was explained by a cooperative globule-tocoil transition at low degrees of ionization, followed by a further expansion of the polymer chain, consequence of the coil-to-expanded conformation transition $[35,36]$. In the case of PAAMe$\operatorname{Py}(150) 55$ and PAAMePy(450)53 polymers, the anomalous behavior was attributed to the formation of hydrophobic microdomains, within the polymer chains, at low $\mathrm{pH}$ values, which lead to an increase of the $\mathrm{p} K_{\mathrm{a}}$ value relative to the unlabeled polymer.

In the case of short-sized chain polymer, the $\mathrm{p} K_{\mathrm{a}}$ value could not be obtained. A decrease of the $I_{B} / I_{C}$ ratio with the $\mathrm{pH}$ was also observed, but due to the experimental error associated to the obtained values, no accurate $\mathrm{p} K_{\mathrm{a}}$ value could be determined. In the case of the labeled PAAMePy polymers the $\mathrm{p} K_{\mathrm{a}}$ value was determined even with for the $2000 \mathrm{~g} / \mathrm{mol} M_{\mathrm{w}}$ polymer. The higher sensitivity of the RRS technique for the pyrene-labeled polymer, when compared with the correspondent unlabeled polymer, is explained by the higher molar extinction coefficient $(\epsilon)$ of the former polymer, which is directly related with the RRS intensity [23].

In dioxane:water mixtures, the $R$ ratio seems, for the unlabeled polymer, and within the experimental error, to display rougher constant values (Fig. 7). However, with the labeled polymer(s) there is a change in the $R$ ratio (see Figs. 4B and 7) which shows that the expansion of the PAA chain, of the PAAMePy polymers, with the increment of the dioxane content in the mixture is, again, due to the presence of hydrophobic groups covalently bound to the PAA chain. In the absence of pyrene groups this effect is not observed and minor changes in the PAA polymer chain are promoted by an increase of the dioxane content in the mixture.

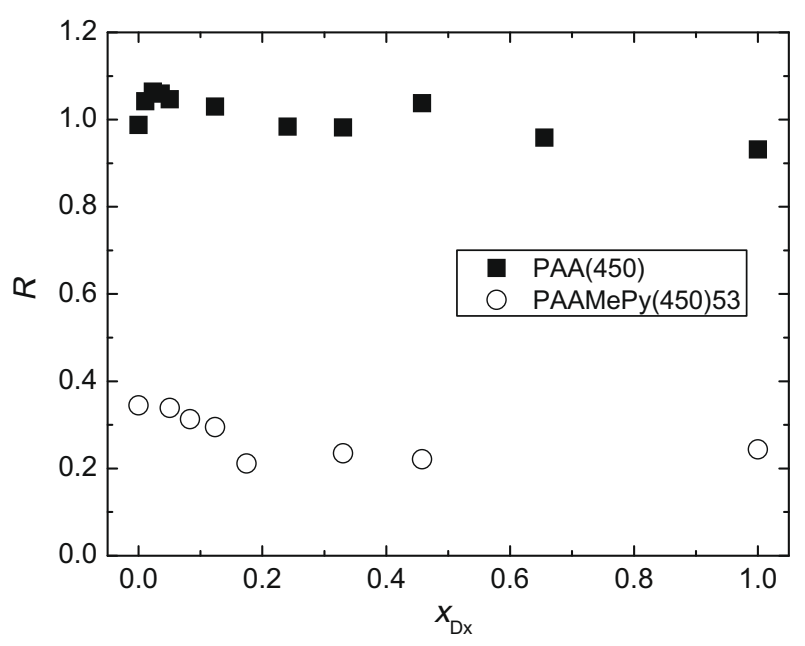

Fig. 7. Plot of the $R$ ratio as a function of the molar fraction of dioxane for the PAA $(450)$ and PAAMePy $(450) 53$ polymers. The $R$ ratio consists in the $I_{\mathrm{B}} / I_{\mathrm{C}}$ and the $I_{\mathrm{B}} /$ $I_{\mathrm{F}}$ ratios for the $\mathrm{PAA}(450)$ and $\mathrm{PAAMePy}(450) 53$ polymers, respectively.

\section{Conclusions}

A RRS study on low and high $M_{\mathrm{w}}$ PAA labeled (with pyrene) and unlabeled polymers was performed showing that identical conclusions could be drawn from those obtained from previous fluorescence investigations. The RRS technique showed to be very useful to compare the behavior of these polymers in solution in the absence and presence of tagged pyrene groups. In the case of the high $M_{\mathrm{w}}$ polymers, the same behavior was found for both the labeled and unlabeled polymers in aqueous solution: predominance of the associative behavior at low $\mathrm{pH}$ (high $I_{\mathrm{RRS}}$ values) and predominance of the repulsive electrostatic repulsions at high $\mathrm{pH}$ (low $I_{\mathrm{RRS}}$ values). For the low $M_{\mathrm{w}}$ polymers the presence of the pyrene probe tagged to the $\operatorname{PAA}(2)$ chain affects their behavior in solution: the unlabeled polymer shows a typical behavior of a polyelectrolyte, i.e., the increase of the $\mathrm{pH}$ leads to an expansion of the polymer chain due to the predominance of repulsive electrostatic interaction between the deprotonated groups, whereas in the case of the labeled polymers the adoption of a "micelle-like" conformation was detected at intermediate $\mathrm{pH}$ values. In dioxane:water mixtures, the dioxane content in the mixture has little effect on the conformation adopted by the unlabeled polymers, whereas in the case of labeled polymers an expansion of the PAAMePy polymers was observed due the increase of the dioxane content of the mixture. The overall study showed that the RRS technique can be easily performed in a spectrofluorimeter (where the steady-state fluorescence experiments are also obtained), prompting for the importance of this approach as a useful and complementary technique for the investigation of macromolecular structures.

\section{Acknowledgements}

Financial support from the Portuguese Science Foundation (FCT) through FEDER and POCI is acknowledged. TC acknowledges the FCT for a PhD grant (SFRH/BD/17852/2004). We thank Dr. Dan Anghel and Dr. Karin Schillén for the PAAMePy(150)55 polymer and Dr. I. Gonçalves, S. Gago and A. Francisco for the help with the synthesis of the PAAMePy(2) and PAAMePy(450) pyrene-labeled polymers.

\section{References}

[1] G.K. Greminger, K.L. Krumel, in: R.L. Davidson (Ed.), Handbook of WaterSoluble Gums and Resins, McGraw-Hill Book Company., New York, 1980.

[2] T. Nishikawa, K. Akiyoshi, J. Sunamoto, J. Am. Chem. Soc. 118 (1996) 6110.

[3] K. Holmberg, B. Jönsson, B. Kronberg, B. Lindman, Surfactants and Polymers in Aqueous Solution, second ed., John Wiley \& Sons Ltd., West Sussex, 2003.

[4] K.G. Shaw, D.P. Leipold, J. Coat. Technol. 57 (1985) 63.

[5] J. Seixas de Melo, T. Costa, A. Francisco, A.L. Maçanita, S. Gago, I.S. Gonçalves, Phys. Chem. Chem. Phys. 9 (2007) 1370.

[6] J. Seixas de Melo, T. Costa, M.D. Miguel, B. Lindman, K. Schillén, J. Phys. Chem. B 107 (2003) 12605.

[7] J.B. Birks, Photophysics of Aromatic Molecules, Wiley, London, 1970.

[8] A. Gilbert, J. Baggott, Essentials of Molecular Photochemistry, Blackwell Scientific Publications, Oxford, 1991.

[9] F.M. Winnik, Chem. Rev. 93 (1993) 587

[10] T. Costa, J. Seixas de Melo, J. Polym. Sci. A Polym. Chem. 46 (2008) 1402.

[11] J. Seixas de Melo, T. Costa, N. Oliveira, K. Schillén, Polym. Int. 56 (2007) 882.

[12] D.C. Dong, M.A. Winnik, Can. J. Chem. Rev. Can. Chim. 62 (1984) 2560.

[13] S. Kanagalingam, C.F. Ngan, J. Duhamel, Macromolecules 35 (2002) 8560.

[14] S. Kanagalingam, J. Spartalis, T.M. Cao, J. Duhamel, Macromolecules 35 (2002) 8571.

[15] D.S. Karpovich, G.J. Blanchard, J. Phys. Chem. 99 (1995) 3951.

[16] K.W. Street, W.E. Acree, Analyst 111 (1986) 1197.

[17] B. Valeur, in: S.G. Schulman (Ed.), Molecular Luminescence Spectroscopy. Methods and Applications, Wiley Interscience, New York, 1993, p. 25.

[18] F.M. Winnik, M.A. Winnik, H. Ringsdorf, J. Venzmer, J. Phys. Chem. 95 (1991) 2583.

[19] C. Cabaleiro-Lago, M. Nilsson, A.J.M. Valente, M. Bonini, O. Soderman, J. Colloid Interface Sci. 300 (2006) 782.

[20] M. Nilsson, C. Cabaleiro-Lago, A.J.M. Valente, O. Soderman, Langmuir 22 (2006) 8663.

[21] A. Sandier, W. Brown, H. Mays, C. Amiel, Langmuir 16 (2000) 1634. 
[22] G.A. Miller, J. Phys. Chem. 82 (1978) 616

[23] R.F. Pasternack, P.J. Collings, Science 269 (1995) 935

[24] S.P. Liu, H.Q. Luo, N.B. Li, Z.F. Liu, W.X. Zheng, Anal. Chem. 73 (2001) 3907.

[25] S. Liu, F. Wang, Z. Liu, X. Hu, A. Yi, H. Duan, Anal. Chim. Acta 601 (2007) 101

[26] X. Long, C. Zhang, J. Cheng, S. Bi, Spectrochim. Acta A 69 (2008) 71.

[27] N.B. Li, H.Q. Luo, S.P. Liu, Spectrochim. Acta A Mol. Biomol. Spectrosc. 60 (2004) 1811

[28] Y. Li, X. Chen, M. Zhang, W. Luo, J. Yang, F. Zhu, Macromolecules 41 (2008) 4873.

[29] B. Chu, Laser Light Scattering, Basic Principles and Practice, second ed., Academic Press Inc., New York, 1991.
[30] T. Costa, M.G. Miguel, B. Lindman, K. Schillén, J. Seixas de Melo, J. Phys. Chem. B 109 (2005) 11478

[31] A.G. Szabo, Fluorescence principles and measurement, in: M.G. Gore (Ed.), Spectrophotometry \& Spectrofluorimetry, Oxford University Press, Oxford, 2000, p. 33.

[32] T. Costa, M.G. Miguel, B. Lindman, K. Schillén, J.C. Lima, J. Seixas de Melo, J. Phys. Chem. B 109 (2005) 3243.

[33] K. Schillén, D.F. Anghel, M.D. Miguel, B. Lindman, Langmuir 16 (2000) 10528

[34] J.W. Lee, S.Y. Kim, S.S. Kim, Y.M. Lee, K.H. Lee, S.J. Kim, J. Appl. Polym. Sci. 73 (1999) 113.

35] J.C. Leyte, M. Mandel, J. Polym. Sci. A Gen. Pap. 2 (1964) 1879.

[36] V. Crescenzi, Adv. Polym. Sci. 5 (1968) 358. 